\title{
PyRice: a Python package for querying Oryza Sativa databases
}

\author{
Quan Do ${ }^{1,3}$, Ho Bich Hai ${ }^{2}$, Pierre Larmande ${ }^{\star 1,3,4}$ \\ ${ }^{1}$ University of Science and Technology of Hanoi (USTH), Hanoi, Vietnam \\ ${ }^{2}$ Institute of Information Technology (IOIT), Vietnam Academy of Science and Technology, Hanoi, Vietnam \\ ${ }^{3}$ DIADE, Univ Montpellier, IRD, Montpellier, France \\ ${ }^{4}$ SouthGreen Bioinformatics Platform, Montpellier, France \\ *To whom correspondence should be addressed.
}

\begin{abstract} sometimes query APIs, which might not always be straightforward for domain experts. The challenge is to to use and produces intuitive results.

Availability and implementation: https://github.com/SouthGreenPlatform/PyRice

Documentation: https://pyrice.readthedocs.io

Contact: pierre.larmande@ird.fr

Licence information: MIT

Supplementary information: Supplementary data are available online.
\end{abstract}

Summary: Currently, gene information available for Oryza sativa species is located in various online heterogeneous data sources. Moreover, methods of access are also diverse, mostly web-based and collect information quickly from these applications and combine it logically, to facilitate scientific research. We developed a Python package named PyRice, a unified programming API to access all supported databases at the same time with consistent output. PyRice design is modular and implements a smart query system which fits the computing resources to optimize the query speed. As a result, PyRice is easy

\section{Introduction}

Rice, a model crop plant, is a major cereal grain widely consumed by a large part of the world's human population, especially in Asia. Since two decades, many digital resources have been developed in rice genomics. However, compared to human genomics, not as much centralized resources and analysis tools are available for rice genomics. Moreover, most of the information currently available are scattered and patchy in nature. Thus, for scientists, the challenge lies in integrating data and finding useful information. In the scope of the project, we aim to build an API to solve the problem of collecting and managing gene and gene products information from different sources. The PyRice package is developed to run remote queries over ten databases and web applications so far. However, PyRice provides generic wrappers to extend this number with more datatypes such as genetics, gene expression and pathways, according (Garg P. et al., 2016) databases classification. Moreover, PyRice uses parallel processing to improve query speed. Furthermore, it indexes results for a fast search and also supports exporting results into different formats.

\section{Materials and Methods}

Information of Oryza sativa genes are published on several open-access databases using different gene annotation models, e.g. RAPDB (Hiroaki et al., 2013), MSU7 or dedicated IDs (i.e. SNP-SEEK (Mansueto et al., 2016) and IC4R (IC4R Project Consortium et al., 2016). PyRice manages a dictionary of ID mapping across databases since each uses either of the two systems RAPDB and MSU7 (e.g. LOC_Os01g01010 = Os01g0100100 while the first ID is from MSU7 and the second is from RAPDB) Supplementary Fig. S1 shows the flowchart of the package. There are two main query types: with genomic coordinates or with a list of gene IDs.

\subsection{Databases}

Currently, PyRice supports 10 databases to query gene information Oryzabase (Kurata et al., 2006), RAPDB, Funricegenes (Yao et al., 2017), Gramene (Tello-Ruiz et al., 2018), IC4R, MSU7 Rice GAP, Rice SNPSEEK, EMBL-EBI Expression Atlas (Papatheodorou et al., 2017) and GWAS Atlas (Tian et al., 2019).

In PyRice, databases information is stored in a unique XML configuration file which allows the users to easily add new databases or update information. In order to help with connection failure due to network or database server's unavailability, some lightweight databases (i.e. SNPSEEK, Oryzabase, RAPDB, GWAS Atlas) are downloaded and cached locally in PyRice after their first query. They are regularly updated within package by built-in function. The PyRice package builds a cross-reference dictionary between three types of index (i.e. MSU7, RAPDB and SNPSEEK) to facilitate information retrieval. Essentially, this means PyRice creates a dictionary of ID mappings with the ones mentioned above, valid them and assign them with internal IDs. It also contains for each gene, ID mappings along with genomic coordinates extracted from SNP-SEEK database. 


\subsection{Parallel processing}

We implemented a smart query system which fits the computing resources and manages the query processing (presented in section 2.3). PyRice runs independently each task in parallel, thus it is able to ignore interrupted tasks due to internet error correction or databases maintenance which may lead to conflict between queries. Moreover, we implemented severa internet client authentication methods in order to avoid server rejections. Since computing resources could have different architectures, PyRice implements 3 parallel options (i.e. multi-processing, multi-threading and the combination of two). Thus, users can choose the number of thread and CPUs depending on their computer resource. In Supplementary File 1 , we evaluated the benefits of using the parallel architectures.

\subsection{Query system}

In PyRice, the query system is based on a database wrapper model. Because online databases usually provide web UI or APIs to query information, we developed a dozen of wrappers for each type of querying methods (e.g. GET, POST), input parameters, and output formats. Furthermore it is possible to configure some filters to keep the necessary attributes Results are indexed by IDs and stored in dictionaries along with databases information (presented in section 2.4). As mentioned previously, Oryza sativa databases use diverse types of IDs to store information. PyRice handles it with a cross-reference dictionary of ID mappings. There are two types of query that is supported in PyRice, based on this dictionary:

- Using IDs: PyRice automatically detects the type of query IDs and maps to those used in the requested database. ID availability check is also conducted before executing the query.

- Using genomic coordinates: These are defined by chromosome number, start and end position.

\subsection{Results}

As presented in section 2.2, PyRice stores results in a dictionary structure (shown in Supplementary File 2). Since gene information exists in several databases, this allows organizing information quickly while saving computing resources. This dictionary is then used to produce final results. Currently, the supported outputs are:

- A summary file is created for each gene found in the initial query. This file gathers all information extracted from the databases and uses the HTML or JSON format for convenient browsing (see Supplementary Fig. S3);

- A spreadsheet stores the resulting gene list along with basic information. PyRice supports output formats of CSV, JSON, HTML, and pickle (see Supplementary Fig. S2).
Pickle format (i.e. .pkl) is a serializing Python object structure which scales up very well with distributed processes, hence can handle a large number of gene queries. PyRice also provides a search and filtering functions for this format, i.e. complex searches using SQL logical operators, i.e. AND, OR, NOT, and result filtering.

\section{Conclusion}

PyRice is a python package that simplifies querying Oryza sativa databases, making it easier to synthesize gene information. PyRice allows multiple processes that help to increase the speed of queries when multicore processors are available. The usage is straightforward, producing intuitive results. It can be easily extended with new databases (see Supplementary File 3), potentially applying to other plants as well.

In the near future, we will extend PyRice to support more databases and new species. Besides, we will provide user support to add new users' databases. To speed up the query process, which is currently parallelized on a single computer, a distributed version of PyRice will be developed. Thus, the new version will use the resources of HPC clusters.

\section{Acknowledgements}

The authors thank the former students, Dang Lam and Vautrin Baptiste who implemented the first prototype of this package.

\section{Funding}

This work has been supported by the CGIAR CRP RICE, IRD DIADE UNIT, The LMI RICE and ICTLab (USTH).

\section{References}

Garg P., Jaiswal P. (2016). Databases and bioinformatics tools for rice research. Current Plant Biology, 7, 39-52

Hiroaki,S., et al. (2013) Rice Annotation Project Database (RAP-DB): an integrative and interactive database for rice genomics. Plant and Cell Physiology, 54.2 (2013), e6-e6.

IC4R Project Consortium, et al. (2016) Information commons for rice (IC4R) Nucleic acids research, 44.D1 (2016), D1172-D1180.

Kurata.N, Yukiko Yamazaki, et al. (2006) Oryzabase. An integrated biological and genome information database for rice. Plant physiology, 140.1 (2006), 12-17.

Mansueto, et al. (2016) Rice SNP-seek database update: new SNPs, indels, and queries. Nucleic acids research, 45(D1), D1075-D1081

Papatheodorou, et al. (2017) Expression Atlas: gene and protein expression across multiple studies and organisms. Nucleic acids research, 46(D1), D246-D251 Tello-Ruiz, et al. (2018) Gramene 2018: unifying comparative genomics and pathway resources for plant research. Nucleic acids research, 46.D1 (2017), D1 181-D1 189. Tian, et al. (2019) GWAS Atlas: a curated resource of genome-wide variant-trait associations in plants and animals. Nucleic Acids Research.

UniProt Consortium. (2018) UniProt: a worldwide hub of protein knowledge. Nucleic acids research, 47.D1 (2018), D506-D515.

Yao, W., et al. (2017) funRiceGenes dataset for comprehensive understanding and application of rice functional genes. Gigascience, 7(1), gix119. 